\title{
Fever During Anti-integrin Therapy: New Immunodeficiency
}

\author{
Eleonora Bonfanti ${ }^{1}$, Christian Bracco ${ }^{1}$, Paolo Biancheri ${ }^{2}$, Andrea Falcetta ${ }^{1}$, Marco Badinella Martini ${ }^{1}$, Remo Melchio ${ }^{1}$ Luigi Fenoglio $^{1}$ \\ ${ }^{1}$ Department of Internal Medicine, "Santa Croce e Carle" Hospital, Cuneo, Italy \\ ${ }^{2}$ Norwich Medical School, University of East Anglia, Norwich Research Park, Norwich, United Kingdom
}

Received: 21/09/2019

Accepted: $14 / 11 / 2019$

Published: $11 / 02 / 2020$

How to cite this article: Bonfanti E, Bracco C, Biancheri P, Falcetta A, Badinella Martini M, Melchio R, Fenoglio L. Fever during anti-integrin therapy: new immunodeficiency. EJCRIM 2020;7: doi:10.12890/2020_001288.

Conflicts of Interests: The Authors declare that there are no competing interests.

This article is licensed under a Commons Attribution Non-Commercial 4.0 License

\section{ABSTRACT}

Background: The causes of inflammatory bowel disease (IBD) have not yet been clearly elucidated, but it is known that genetic susceptibility, altered gut microbiota and environmental factors are all involved, and that a combination of these factors causes an inappropriate immune response, resulting in impaired intestinal barrier function. With regard to the treatment of IBD, the use of conventional immunosuppressive drugs has been complemented by more specific therapeutic agents, including biological drugs. Systemic immune suppression is a risk factor for cytomegalovirus (CMV) infection, which is associated with considerable morbidity and mortality in immunocompromised hosts.

Case Report: A 33-year-old male patient was admitted to our medical unit complaining of a 10-day history of fever, fatigue and headache. He had been suffering from ulcerative colitis and primary sclerosing cholangitis for five years and was currently being treated with azathioprine and vedolizumab. In the past he had already taken infliximab, adalimumab and golimumab without any clinical response. After the exclusion of systemic infectious diseases, his serology was consistent with a primary CMV infection. This was successfully treated with intravenous ganciclovir therapy.

Conclusion: Vedolizumab is an anti-integrin biological agent approved for IBD treatment. Its gut-selective mechanism of action would appear to increase its safety profile, however data on this are still limited. Moreover, it should always be remembered that IBD patients have an increased risk of CMV infection, both primary and reactivation, because of their concurrent immunosuppression.

\section{LEARNING POINTS}

- It is important to consider CMV infection (primary and reactivation) in patients affected by IBD.

\section{KEYWORDS}

Vedolizumab, cytomegalovirus, ulcerative colitis

\section{INTRODUCTION}

During the past two decades, the treatment of IBD has been revolutionised with conventional treatments complemented by the advent of biological drugs that target specific molecules of inflammation-related pathways, to induce intestinal mucosal healing and change the progression of the disease.

Anti-tumour necrosis factor (TNF) agents were the first biologics used to treat IBD, however both the first- and second-generation TNFantagonists failed to show an effect across all IBD patients: $10 \%-30 \%$ were primary non-responders, while loss of response was seen in $23 \%$ $46 \%{ }^{[1]}$. More recently, newer biological drugs, called anti-integrins, have been developed and used successfully. These prevent lymphocyte recruitment to the site of inflammation by blocking the interaction between integrins (cell surface proteins expressed by circulating immune cells) and cell adhesion molecules (CAMs), such as MAdCAM-1, which is expressed on the endothelium of intestinal mucosal blood vessels. 
Vedolizumab can be used in moderate-severe IBD patients who did not respond or were intolerant to first- and second-line therapies. Vedolizumab prevents $\alpha 4 \beta 7$-integrin on lymphocyte membrane from binding to its ligand MAdCAM-1: this gut-selective mechanism prevents lymphocyte recruitment to the inflamed IBD mucosa ${ }^{[2]}$.

\section{CASE REPORT}

A 33-year-old male patient was admitted to our medical unit because of a 10 -day history of fever (up to $39^{\circ} \mathrm{C}$ ), not responsive to paracetamol. $\mathrm{He}$ also complained of fatigue and right frontal headache, associated with nausea and photophobia. He reported 5 bowel movements per day on average, with mucus but no previous bleeding. For the past five years had been suffering from ulcerative colitis (UC) and primary sclerosing cholangitis. His whole colon was involved and he had already taken infliximab, adalimumab and golimumab, without any clinical response. He had never been exposed to steroid therapy. At the time of admission, he had been under treatment with azathioprine (50 $\mathrm{mg}$ bid) and vedolizumab (300 mg every 8 weeks) for four months with a good clinical response and no signs of flares on laboratory and endoscopic evaluations. Both focal neurological and meningeal signs were negative. Chest X-ray, head CT, brain MRI and eye examinations were all unremarkable. On the neurologist's advice, lumbar puncture was not performed as his headache was interpreted as a symptom of systemic infection. He was started on empirical antibiotic treatment with intravenous (IV) ceftriaxone and oral levofloxacin. Blood tests showed mild anaemia and thrombocytopenia without lymphocytosis.

Urine and blood cultures were negative, and echocardiogram did not show any evidence of endocarditis. Abdominal ultrasound showed splenomegaly and PET/CT imaging was compatible with an IBD flare. Faecal calprotectin was markedly increased (> 2000 mcg/g), while stool cultures were negative for intestinal infections, including Clostridium difficile. Furthermore, diagnostic tests for malaria parasites, Brucella, toxoplasma, Borrelia, Rickettsiae, leishmaniasis, beta-D-glucan and HIV were negative, as were other tests: quantiferon, monotest and antistreptolysin O titer (ASLOT).

A few days later his serology was consistent with a primary $C M V$ infection: IgG were absent $(<5 \mathrm{U} / \mathrm{ml})$, while IgM were higher than normal $(117 \mathrm{U} / \mathrm{ml})$ and CMV DNA levels displayed an incremental trend (from 1739 to $2819 \mathrm{Ul} / \mathrm{m})$. Since the patient was also immunosuppressed, IV injections of ganciclovir ( $5 \mathrm{mg} / \mathrm{kg}$ ) twice a day for two weeks were administered and azathioprine was stopped. His fever disappeared and his general conditions rapidly improved, so the patient was discharged. He continued with oral ganciclovir therapy ( $5 \mathrm{mg} / \mathrm{kg}$ bid) for 14 days. He also took mesalazine (800 mg bid) until the next colonoscopy.

\section{DISCUSSION}

Cytomegalovirus (CMV) is a ubiquitous virus and its seroprevalence in the general population ranges from 40 to $100 \%$. Primary CMV infection in the immunocompetent host is often asymptomatic, while in immunocompromised individuals it can be associated with considerable morbidity and mortality.

This opportunistic pathogen is more commonly detected in medically refractory UC than in Crohn's disease because of their different immunological backgrounds ${ }^{[3]}$. The prevalence of CMV reactivation in moderate-to-severe colitis has been reported as between $21 \%$ and $34 \%$, and the prevalence of CMV in glucocorticoid refractory colitis is greater than $30 \%{ }^{[4]}$. A recent study revealed that the CMV seroprevalence rate (primary infections) in European IBD patients is probably lower than previously thought ${ }^{[5]}$. IBD patients can be considered at increased risk of CMV infection, both primary and reactivation, given their immune dysregulation, reduced barrier function, possible malnutrition and possible immunosuppressive therapy ${ }^{[4]}$. Clinical practice guidelines issued by the Italian Group for the study of IBDs state that thiopurine-treated IBD patients have a 3-fold increased risk of opportunistic infections such as CMV, while IBD patients receiving thiopurine and steroids have a 5 -fold increased risk ${ }^{[6]}$. Furthermore, a recent meta-analysis found that patients treated with biologics had a moderately increased risk of any infection (OR 1,19; 95\% C.I. 1,10-1,29) and a significantly increased risk of opportunistic infections (OR 1,90; 95\% C.I. 1,21-3,01) ${ }^{[7]}$. Other studies analyzed vedolizumab effectiveness and safety; in one of them, active smoking and concomitant use of immunosuppressive agents and corticosteroids were independently associated with infections ${ }^{[8]}$. Currently, in IBD patients, ganciclovir administration is indicated for primary CMV infection associated with exacerbation of colitis, and also when CMV is detected in colonic mucosa or in severe steroid-resistant colitis patients. For example, a recent case report showed that vedolizumab seems to be safe in patients with active UC and colonic CMV reactivation as long as concurrent antiviral treatment is started ${ }^{[9]}$.

\section{CONCLUSION}

When we encounter fever in IBD patients, it is important to consider the possibility of CMV infection, both primary and reactivation.

Very little is known about how to manage CMV infections in patients who are being treated with anti-integrin drugs and we need more data 


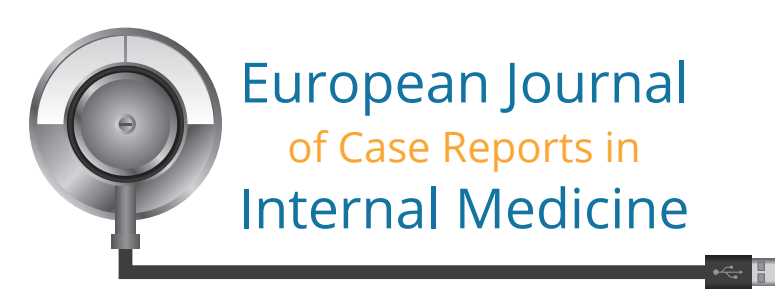

to understand how biological drugs affect the patient immune system.

Vedolizumab therapy seems to be associated with a low side-effects profile, but further real-life studies need to be conducted to improve our knowledge about its safety, especially when it is taken at the same time as corticosteroids.

\section{REFERENCES}

1. Roda G, Jharap B, Neeraj N, Colombel JF. Loss of response to anti-TNFs: definition, epidemiology, and management. Clin Transl Gastroenterol 2016 ; 7:e135.

2. Wyant T, Leach T, Sankoh S, Wang Y, Paolino J, Pasetti MF, et al. Vedolizumab affects antibody responses to immunisation selectively in the gastrointestinal tract: randomised controlled trial results. Gut 2015;64:77-83.

3. Strober W, Fuss IJ. Proinflammatory cytokines in the pathogenesis of inflammatory bowel diseases. Gastroenterology 2011:140:1756-1767.

4. Beswick L, Ye B, Van Langenberg DR. Toward an algorithm for the diagnosis and management of CMV in patients with colitis. Inflamm Bowel Dis 2016;22:2966-2976.

5. Rowan C, Judge C, Cannon MD, Cullen G, Mulcahy HE, Ryan E, et al. Severe symptomatic primary CMV infection in inflammatory bowel disease patients with low population seroprevalence. Gastroenterol Res Pract 2018;2018:1029401

6. Aratari A, Bossa F, Cappello M, Cassinotti A, Chiriatti A, Fiorino G, et al. Safety of treatments for inflammatory bowel disease: Clinical practice guidelines of the Italian Group for the Study of Inflammatory Bowel Disease (IG-IBD). Dig Liver Dis 2017;49:338-358.

7. Bonovas S, Fiorino G, Allocca M, Lytras T, Nikolopoulos GK, Peyrin-Biroulet L, et al. Biologic therapies and risk of infection and malignancy in patients with inflammatory bowel disease: a systematic review and network meta-analysis. Clin Gastroenterol Hepatol 2016;14:1385-1397.

8. Meserve J, Aniwan S, Koliani-Pace JL, Shashi P, Weiss A, Faleck D, et al. Retrospective analysis of safety of vedolizumab in patients with inflammatory bowel diseases. Clin Gastroenterol Hepatol 2019;17:1533-1540.

9. Hommel C, Pillet S, Rahier J-F. Comment on: 'Resolution of CMV infection in the bowel on vedolizumab therapy.' J Crohn's Colitis 2019 pii: jjz108. doi: 10.1093/ecco-jcc/jjz108. 